\title{
Definable hereditary families in the projective hierarchy
}

\author{
by
}

\author{
Rana Barua and V. V. Srivatsa (Calcutta)
}

\begin{abstract}
We show that if $\mathcal{F}$ is a hereditary family of subsets of $\omega^{\omega}$ satisfying certain definable conditions, then the $\Delta_{1}^{1}$ reals are precisely the reals $\alpha$ such that $\{\beta$ : $\left.\alpha \in \Delta_{1}^{1}(\beta)\right\} \notin \mathcal{F}$. This generalizes the results for measure and category. Appropriate generalization to the higher levels of the projective hierarchy is obtained under Projective Determinacy. Application of this result to the $Q_{2 n+1}$-encodable reals is also shown.
\end{abstract}

0. Introduction. In his seminal paper [1], Kechris obtained a number of measure-theoretic and category-theoretic results that respectively seem to depend on the properties of measure and category. In another direction, he obtained a basis theorem for "large" $\pi_{2 n+1}^{1}$ sets (under PD) that works for both measure and category. This is obtained by formulating the basis theorem in terms of $\sigma$-ideals satisfying certain definable conditions (members of the $\sigma$-ideals are regarded as small sets). In much the same spirit, we show that some of the results in [1] depend purely on definable conditions. We work with the more general definable hereditary families, i.e. definable families of subsets of reals closed under set inclusion. That these results hold for hereditary families is of great significance as we shall see later. Our main result is the characterization of $\mathcal{D}_{1}^{1}$, the set of $\Delta_{1}^{1}$ reals and, at the higher levels, of $Q_{2 n+1}$ under PD. We prove:

$\mathcal{D}_{1}^{1}$ is precisely the set of reals $\alpha$ such that $\left\{\beta: \alpha \in \Delta_{1}^{1}(\beta)\right\} \notin \mathcal{J}$, for any $\pi_{1}^{1}$ computable hereditary family $\mathcal{J}$ (see the definition below).

The proof of this fact is soft and easily generalizable. Elementary uses of the $Q$-theory of Kechris-Martin-Solovay [4] yield an analogous characterization of $Q_{2 n+1}$ under PD. The following seems new even for measure:

$$
\alpha \in Q_{2 n+1} \leftrightarrow\left\{\beta: \alpha \leq_{2 n+1}^{Q} \beta\right\} \notin \mathcal{J},
$$

for any $\pi_{2 n+1}^{1}$ computable hereditary family $\mathcal{J}$. The corresponding statement for $\Delta_{2 n+1}^{1}$ is false in this generality. 
The fact that such results hold for hereditary families is of significance. While we know of no non-trivial $\pi_{1}^{1}$ (resp. $\pi_{2 n+1}^{1}$ ) computable $\sigma$-ideals other than the measure and category ones, interesting examples of $\pi_{1}^{1}$ (resp. $\pi_{2 n+1}^{1}$ ) computable hereditary families can be obtained by natural dualization of any $\Sigma_{1}^{1}$ (resp. $\quad \Sigma_{2 n+1}^{1}$ ) computable hereditary families. This yields analogous results for $\Sigma_{1}^{1}$ (resp. $\Sigma_{2 n+1}^{1}$ ) computable hereditary families.

At the higher levels, a new example emerges in the form of the Ramsey ideal. While this is not $\Sigma_{1}^{1}$ computable, for $n \geq 1$, by a result of Kechris [3], this is $\Sigma_{2 n+1}^{1}$ computable under PD. In view of the remarks made above, the characterization quoted earlier applies to yield a different proof of the following result of Kechris [3] on encodable reals:

the $Q_{2 n+1}$-encodable reals are precisely the elements of $Q_{2 n+1}$.

1. The main results. Our basic theory is $\mathrm{ZF}+\mathrm{DC}$. Additional axioms like PD (projective determinacy), $\operatorname{Det}\left(\boldsymbol{\Delta}_{2 n}^{1}\right)$ (determinacy of $\boldsymbol{\Delta}_{2 n}^{1}$ games) would be explicitly mentioned. Our notation and terminology are as in Moschovakis [5]. For our results at the higher levels we assume familiarity with Kechris-Martin-Solovay memoir on $Q$-theory [4].

We now make the following formal definition.

1.1. Definition. Let $\mathcal{I}$ be a family of subsets of reals and let $\Gamma$ be a pointclass. We say that $\mathcal{I}$ is $\Gamma$ computable if for every $B \in \Gamma, B \subseteq X \times \omega^{\omega}$, $X=\left(\omega^{\omega}\right)^{k} \times \omega^{l}$, the set

$$
B^{\Delta}=\left\{x \in X: B_{x} \notin \mathcal{I}\right\}
$$

is in $\Gamma$, where $B_{x}=\{\beta:(x, \beta) \in B\}$.

1.2. Definition. A family $\mathcal{I}$ is said to be hereditary if

$$
B \in \mathcal{I} \& A \subseteq B \rightarrow A \in \mathcal{I} .
$$

We shall need the following folklore type result which we have obtained by dualizing the effective analogue of a result of Piątkiewicz (cf. [6]).

1.3. Lemma. Let $\Gamma$ be a Spector pointclass and $\mathcal{F}$ a $\Gamma$ computable, hereditary family of subsets of $\omega^{\omega}$. Then for every $\Gamma$ set of reals $A \notin \mathcal{F}$ there is $a \Delta=\Gamma \cap \neg \Gamma$ set $B \subseteq A$ such that $B \notin \mathcal{F}$.

Proof. Fix a $\Gamma$-norm $\phi$ on $A$. Suppose $A$ is not $\Delta$. Let

$$
C=\left\{\alpha:\left\{\beta<_{\phi}^{*} \alpha\right\} \in \mathcal{F}\right\} .
$$

Since $\mathcal{F}$ is $\Gamma$ computable, $C$ is in $\neg \Gamma$; and since $A \notin \mathcal{F}, C \subseteq A$. Moreover,

$$
C(\alpha) \& \phi(\beta) \leq \phi(\alpha) \rightarrow C(\beta) \text {. }
$$

Since $A$ is not $\neg \Gamma$ we have $C \varsubsetneqq A$. Hence there is $\alpha_{0} \in A$ such that $C=\left\{\alpha: \alpha<_{\phi}^{*} \alpha_{0}\right\}$. Since $\alpha_{0} \notin C$, it follows that $\left\{\beta: \beta<_{\phi}^{*} \alpha_{0}\right\}=C \notin \mathcal{F}$. Let $B$ be a $\Delta$ set such that $C \subseteq B \subseteq A$. This $B$ does the job. 
We now prove

1.4. ThEOREM. Let $\mathcal{F} \neq \emptyset$ be a hereditary family of subsets of reals not containing $\omega^{\omega}$. Then the following hold:

(a) If $\mathcal{F}$ is $\pi_{1}^{1}$ computable, then

$$
\alpha \in \mathcal{D}_{1}^{1} \leftrightarrow\left\{\beta: \alpha \in \Delta_{1}^{1}(\beta)\right\} \notin \mathcal{F},
$$

where $\mathcal{D}_{1}^{1}$ is the set of all $\Delta_{1}^{1}$ reals.

(b) Assume $\operatorname{Det}\left(\boldsymbol{\Delta}_{2 n}^{1}\right), n \geq 1$, and $\mathcal{F}$ is $\pi_{2 n+1}^{1}$ computable. Then

$$
\alpha \in Q_{2 n+1} \leftrightarrow\left\{\beta: \alpha \leq_{2 n+1}^{Q} \beta\right\} \notin \mathcal{F} .
$$

Proof. We shall prove (b); the proof of (a) is analogous.

If $\alpha$ is $Q_{2 n+1}$ then clearly $\left\{\beta: \alpha \leq_{2 n+1}^{Q} \beta\right\}=\omega^{\omega} \notin \mathcal{F}$. For the converse, first observe that if $\alpha$ is a $\pi_{2 n+1}^{1}(\delta)$ singleton, i.e. $\{\alpha\}$ is $\pi_{2 n+1}^{1}(\delta)$, then the set $E[\alpha] \stackrel{\text { def }}{=}\left\{\beta: \alpha \leq_{2 n+1}^{Q} \beta\right\}$ is easily seen to be $\pi_{2 n+1}^{1}(\delta)$. If, furthermore, $E[\alpha] \notin \mathcal{F}$, then by Lemma 1.3 (with $\Gamma=\pi_{2 n+1}^{1}(\delta)$ ), since it can be checked that $\mathcal{F}$ is $\pi_{2 n+1}^{1}(\delta)$ computable, there is a $\Delta_{2 n+1}^{1}(\delta)$ set $B \subseteq E[\alpha]$ such that $B \notin \mathcal{F}$. So $\alpha \leq_{2 n+1}^{Q} \beta$ for all $\beta \in B$. But then

$$
\alpha(n)=m \leftrightarrow(\forall \beta)\left[\beta \in B \rightarrow\left(\exists \gamma \leq_{2 n+1}^{Q} \beta\right)(\gamma \in\{\alpha\} \& \gamma(n)=m)\right] .
$$

This shows that the graph of $\alpha$ is $\pi_{2 n+1}^{1}(\delta)$ and hence $\alpha$ is $\Delta_{2 n+1}^{1}(\delta)$.

Hence the set

$$
G=\left\{\alpha:\left\{\beta: \alpha \leq_{2 n+1}^{Q} \beta\right\} \notin \mathcal{F}\right\}
$$

contains no non-trivial $\pi_{2 n+1}^{1}(\delta)$ singleton for any $\delta$. Also since $\mathcal{F}$ is $\pi_{2 n+1}^{1}$ computable, $G$ is $\pi_{2 n+1}^{1}$. We now claim that $G$ is thin, i.e. contains no perfect set. If not, there is a recursive-in- $\delta$, one-one function $g: 2^{\omega} \rightarrow G$ for some $\delta$. But $2^{\omega}$ contains a non-trivial $\pi_{2 n+1}^{1}(\delta)$ singleton and so $G$ contains a non-trivial $\pi_{2 n+1}^{1}(\delta)$ singleton. But this is not possible. So $G$ is thin and consequently $G \subseteq C_{2 n+1}$, the largest thin $\pi_{2 n+1}^{1}$ set (cf. [2]). Since $G$ is closed under $\leq_{2 n+1}$ and $G \supseteq Q_{2 n+1}$ contains no non-trivial $\pi_{2 n+1}^{1}$ singleton, $G$ must be the set of $Q_{2 n+1}$ reals (see [4;6.3]). This completes the proof.

1.5. Remark. If $\mathcal{F}$ is the $\sigma$-ideal of meagre sets or of Lebesgue null sets, then the analogue of (b) for $\Delta_{2 n+1}^{1}$ degrees holds (cf. [1]). However, it cannot hold for all $\left(\pi_{2 n+1}^{1}\right.$ computable $) \sigma$-ideals. To see this, let $\mathcal{J}$ be the $\sigma$-ideal of all sets disjoint from $Q_{2 n+1}$. Since $Q_{2 n+1}$ is $\pi_{2 n+1}^{1}$-bounded, $\mathcal{J}$ is easily seen to be $\pi_{2 n+1}^{1}$ computable. Now observe that, trivially, for any $\alpha \in Q_{2 n+1},\left\{\beta: \alpha \in \Delta_{2 n+1}^{1}(\beta)\right\} \notin \mathcal{J}$. And so the following is false:

$$
\alpha \in \mathcal{D}_{2 n+1}^{1} \leftrightarrow\left\{\beta: \alpha \in \Delta_{2 n+1}^{1}(\beta)\right\} \notin \mathcal{J} ;
$$

since for $n \geq 1, Q_{2 n+1}-\mathcal{D}_{2 n+1}^{1} \neq \emptyset$. 
Now suppose $\mathcal{F}$ is a $\Sigma_{2 n+1}^{1}$ computable hereditary family for any $n \in \omega$. Put $\mathcal{F}^{0}=\left\{A \subseteq \omega^{\omega}: A^{c} \notin \mathcal{F}\right\}$. Then it is easy to see that $\mathcal{F}^{0}$ is a $\pi_{2 n+1}^{1}$ computable hereditary family. Applying Theorem 1.4 to $\mathcal{F}^{0}$ and unfolding the definition of $\mathcal{F}^{0}$ one obtains:

1.6. TheOREM. Let $\mathcal{F} \neq \emptyset$ be a hereditary family of subsets of reals not containing $\omega^{\omega}$.

(a) If $\mathcal{F}$ is $\Sigma_{1}^{1}$ computable, then

$$
\alpha \in \mathcal{D}_{1}^{1} \leftrightarrow\left\{\beta: \alpha \notin \Delta_{1}^{1}(\beta)\right\} \in \mathcal{F} .
$$

(b) Assume $\operatorname{Det}\left(\boldsymbol{\Delta}_{2 n}^{1}\right)$ and $n \geq 1$. If $\mathcal{F}$ is $\Sigma_{2 n+1}^{1}$ computable, then

$$
\alpha \in Q_{2 n+1} \leftrightarrow\left\{\beta: \neg\left(\alpha \leq_{2 n+1}^{Q} \beta\right)\right\} \in \mathcal{F} .
$$

As an immediate consequence of Theorem 1.4 we have the following

1.7. Theorem. Let $\mathcal{F}$ be as above.

(a) Suppose $\mathcal{F}$ is $\pi_{1}^{1}$ computable. Then $\left\{\alpha: \omega_{1}^{c k}<\omega_{1}^{\alpha}\right\} \in \mathcal{F}$.

(b) Assume $\operatorname{Det}\left(\boldsymbol{\Delta}_{2 n}^{1}\right), n \geq 1$ and suppose $\mathcal{F}$ is $\pi_{2 n+1}^{1}$ computable. Then

$$
\left\{\alpha: k_{2 n+1}<k_{2 n+1}(\alpha)\right\} \in \mathcal{F},
$$

where $\alpha \rightarrow k_{2 n+1}(\alpha)$ is the ordinal assignment for $Q_{2 n+1}$ degrees (as in Sec. 14 of [4]).

Pr o of. We prove (b) since the proof of (a) is similar. First observe that by 14.8 of [4],

$$
k_{2 n+1}<k_{2 n+1}(\alpha) \leftrightarrow y_{2 n+1}^{0} \in \Delta_{2 n+1}^{1}(\alpha),
$$

where $y_{2 n+1}^{0}$ is the first non-trivial $\pi_{2 n+1}^{1}$ singleton. Thus, if $\left\{\alpha: k_{2 n+1}<\right.$ $\left.k_{2 n+1}(\alpha)\right\} \notin \mathcal{F}$, then by Theorem $1.4(\mathrm{~b}), y_{2 n+1}^{0} \in Q_{2 n+1}$. But $Q_{2 n+1}$ contains no non-trivial $\pi_{2 n+1}^{1}$ singleton. This proves the assertion in (b).

Arguing as in 1.6 above we obtain

1.8. Corollary. Let $\mathcal{F}$ be as above.

(a) If $\mathcal{F}$ is $\Sigma_{1}^{1}$ computable, then $\left\{\alpha: \omega_{1}^{c k}=\omega_{1}^{\alpha}\right\} \notin \mathcal{F}$.

(b) Assume $\operatorname{Det}\left(\boldsymbol{\Delta}_{2 n}^{1}\right)$ and $n \geq 1$. If $\mathcal{F}$ is $\Sigma_{2 n+1}^{1}$ computable, then $\left\{\alpha: k_{2 n+1}=k_{2 n+1}(\alpha)\right\} \notin \mathcal{F}$.

1.9. Remark. Notice that 1.8 above is a generalization of the Gandy Basis Theorem (cf. [4; Sec. 14.9]). For if $A$ is $\Sigma_{2 n+1}^{1}$ and non-empty, the $\sigma$ ideal consisting of sets disjoint from $A$ is $\Sigma_{2 n+1}^{1}$ computable. The statement (b) above for this ideal asserts that there is $\alpha \in A$ with $k_{2 n+1}=k_{2 n+1}(\alpha)$. 
2. Application. The above characterization of $Q_{2 n+1}$ for a suitable $\mathcal{F}$ yields simplifications of a result of Kechris on the $Q_{2 n+1}$-encodable reals (see [3]). The relevant definitions are as follows.

2.1. Definition. Let $\leq_{\mathrm{r}}$ be any notion of reducibility among reals (like for instance Turing reducibility $\leq_{\mathrm{T}}$ ). Let $[\omega]^{\omega}$ denote the set of all infinite subsets of $\omega$. A real $\alpha$ is said to be $\leq_{\mathrm{r}}$ encodable iff

$$
\forall X \in[\omega]^{\omega} \exists Y \in[\omega]^{\omega}\left(Y \subseteq X \text { and } \alpha \leq_{\mathrm{r}} Y\right) .
$$

When $\leq_{\mathrm{r}}=\leq_{\mathrm{T}}, \alpha$ is said to be recursively encodable; when $\leq_{\mathrm{r}}=\leq_{n}, \alpha$ is said to be $\Delta_{n}^{1}$-encodable; when $\leq_{\mathrm{r}}=\leq_{2 n+1}^{Q}, \alpha$ is said to be $Q_{2 n+1}$-encodable.

From now on infinite subsets of $\omega$ will be denoted by $X, Y, \ldots$

2.2. Definition. Let $s$ be a finite set of natural numbers and $X$ an infinite one. The pair $\langle s, X\rangle$ is said to be a condition if $\max (s)<\min (X)$. The Ellentuck neighbourhood $(s, X)$ consists of all $Y \in[\omega]^{\omega}$ such that $s \subseteq$ $Y \subseteq s \cup X$. A subset $A$ of $[\omega]^{\omega}$ is said to be Ramsey null if for every $X$ there exists $Y \subseteq X$ such that $A \cap(\emptyset, Y)=\emptyset$. It is said to be completely Ramsey null if for each Ellentuck neighbourhood $(s, X)$ there exists $Y \subseteq X$ such that $A \cap(s, Y)=\emptyset$.

Let $\mathcal{J}_{1}, \mathcal{J}_{2}$ denote the families of Ramsey null and completely Ramsey null sets respectively. Clearly $\mathcal{J}_{i}$ is hereditary and it is well known that $\mathcal{J}_{i}$ is a $\sigma$-ideal, $i=1,2$.

2.3. Lemma (Assume PD for $n \geq 1$ ). Let $\leq_{\mathrm{r}}$ be $\leq_{2 n+1}$ or $\leq_{2 n+1}^{Q}$. Then $\alpha$ is $\leq_{\mathrm{r}}$-encodable iff $\left\{X: \neg\left(\alpha \leq_{\mathrm{r}} X\right)\right\} \in \mathcal{J}_{1}$ iff $\left\{X: \neg\left(\alpha \leq_{\mathrm{r}} X\right)\right\} \in \mathcal{J}_{2}$.

Proof. Suppose $\alpha$ is $\leq_{\mathrm{r}}$-encodable. Fix $Y$. Now $\left\{X: \neg\left(\alpha \leq_{\mathrm{r}} X\right)\right\}$ is projective, and therefore has the Ramsey property (under PD for $n \geq 1$ ). So, there is $Y^{\prime} \subseteq Y$ such that either $\left(\emptyset, Y^{\prime}\right) \subseteq\left\{X: \alpha \leq_{\mathrm{r}} X\right\}$ or $\left(\emptyset, Y^{\prime}\right) \subseteq\{X$ : $\left.\neg\left(\alpha \leq_{\mathrm{r}} X\right)\right\}$. The second alternative cannot hold since $\alpha$ is $\leq_{\mathrm{r}}$-encodable. But this shows that $\left\{X: \neg\left(\alpha \leq_{\mathrm{r}} X\right)\right\} \in \mathcal{J}_{1}$. The other implications are similar or easier.

2.4. Remark (Failure of computations for the Ramsey ideals). For $\sigma$-ideals $\mathcal{J}$, the $\pi_{1}^{1}$ computability condition almost appears to imply the countable chain condition (c.c.c.) for Borel $/ \mathcal{J}$. Be that as it may, the c.c.c. fails in a very strong form for the Ramsey (and completely Ramsey) ideals. To see this notice that the canonical family of almost disjoint sets in $2^{\omega}$ yields a $\Delta_{1}^{1}$ perfect set $P$ (of codes) of almost disjoint sets. These must correspond to disjoint Ellentuck neighbourhoods. It is not hard to see from here that the $\pi_{1}^{1}$ computability condition must fail (this was also observed independently by B. V. Rao). An identical argument applies to $\pi_{2 n+1}^{1}$, for all $n \geq 1$. 
The $\Sigma_{1}^{1}$ computability condition also fails for the families $\mathcal{J}_{1}$ and $\mathcal{J}_{2}$. This is because by Solovay $([7])$, there is a $\Delta_{1}^{1}$-encodable real which is not $\Delta_{1}^{1}$. If $\alpha$ is such a real, then by Lemma 2.3, $\left\{X: \alpha \notin \Delta_{1}^{1}(X)\right\}$ is completely Ramsey null. But if the ideal $\mathcal{J}_{2}$ were $\Sigma_{1}^{1}$ computable, then Theorem 1.6 would apply to yield $\alpha \in \Delta_{1}^{1}$. Contradiction! The same argument works for $\mathcal{J}_{1}$.

In contrast to the above we will now observe that, under $\mathrm{PD}, \mathcal{J}_{1}$ is $\Sigma_{2 n+1}^{1}$ computable for $n \geq 1$. This follows easily from the following result of Kechris [3], when relativized. If $A$ is $\pi_{2 n+1}^{1}$, then either there is a set $Y$ such that $(\emptyset, Y) \cap A=\emptyset$ or there is a $\Delta_{2 n+1}^{1}$ set $Y$ such that $(\emptyset, Y) \subseteq A$.

Proposition 2.5. Assume $n \geq 1$ and PD. Then the Ramsey ideal $\mathcal{J}_{1}$ is $\Sigma_{2 n+1}^{1}$ computable.

Proof. For any $\Sigma_{2 n+1}^{1}(\alpha)$ set $P$,

$P$ is Ramsey null $\leftrightarrow(\forall X)(\exists Y \subseteq X)[(\emptyset, Y) \cap P=\emptyset]$

$$
\leftrightarrow(\forall X) \exists Y \in \Delta_{2 n+1}^{1}(\langle\alpha, X\rangle)[Y \subseteq X \&(\emptyset, Y) \cap P=\emptyset] ;
$$

the last equivalence holding by an application of Kechris' result relativized. From this it follows easily that $\mathcal{J}_{1}$ is $\Sigma_{2 n+1}^{1}$ computable.

The following is now immediate.

2.6. TheOrem (Kechris). Assume PD and $n \geq 1$. Then the $Q_{2 n+1}$ encodable reals are precisely the $Q_{2 n+1}$ reals.

Proof. If $\alpha \in Q_{2 n+1}$, then clearly $\alpha$ is $Q_{2 n+1}$-encodable. Conversely, if $\alpha$ is $Q_{2 n+1}$-encodable then, by Lemma 2.3, $\left\{X: \neg\left(\alpha \leq_{2 n+1}^{Q} X\right)\right\} \in \mathcal{J}_{1}$. Since by Proposition 2.5, $\mathcal{J}_{1}$ is $\Sigma_{2 n+1}^{1}$-encodable, an application of Theorem 1.6 yields $\alpha \in Q_{2 n+1}$.

Remark. The above proof is different from the one in Kechris [3] and while it does not seem to be technically simpler, it is perhaps of some conceptual significance.

\section{References}

[1] A. S. Kechris, Measure and category in effective descriptive set theory, Ann. Math. Logic 5 (1973), 337-384.

[2] - The theory of countable analytical sets, Trans. Amer. Math. Soc. 202 (1975), 259-267.

[3] - Effective Ramsey theorems in the projective hierarchy, in: Proceedings of the Herbrand Symposium, J. Stern (ed.), North-Holland, Amsterdam 1982.

[4] A. S. Kechris, D. A. Martin and R. M. Solovay, Introduction to Q-theory, in: Cabal Seminar 79-81 (Proceedings, Caltech-UCLA Logic Seminar, 1979-81), Lecture Notes in Math. 1019, Springer, 1983, 199-281.

[5] Y. N. Moschovakis, Descriptive Set Theory, North-Holland, Amsterdam 1980. 
[6] L. Piạtkiewicz, A remark about separation of K-analytic sets in the product spaces, Proc. Amer. Math. Soc. 93 (1985), 363-366.

[7] R. M. Solovay, Hyperarithmetically encodable sets, Trans. Amer. Math. Soc. 239 (1978), 99-122.

STAT-MATH DIVISION

INDIAN STATISTICAL INSTITUTE

203 B.T. ROAD

CALCUTTA 700035, INDIA 